\title{
Efficacy of different prediabetes program models in improving clinical outcomes in people with prediabetes
}

\section{Abstract}

Background: Educational programs in general seem to have a clinically significant beneficial effect among the T2DM population in terms of improved glycaemic control. However, few evaluations of interventions to delay or prevent type 2 diabetes mellitus (T2DM) in Saudi Arabia (SA) have been undertaken.

Objective: The present study evaluates for the first time, the differences in the effectiveness of the different educational programs [intensive lifestyle modification (Group Education Program, GEP), supervised education through social media (WhatsApp Education Group, WEP) and standard care via PHCCs (Control Group, CG)], among Saudi females with pre-diabetes.

Methods: This was a 6-month, multi-center, 3-arm cluster, randomised, controlled (1:1:1), multi-intervention study conducted from July 2018 until March of 2019 in Riyadh, SA. A total of 1140 females from SA were cluster randomised equally to three groups, out of which only $253[\mathrm{~N}=100 \mathrm{GEP}, \mathrm{N}=84 \mathrm{WEP}$ and $\mathrm{N}=69 \mathrm{CG}]$ received intervention. Participants completed questionnaires including demographic, dietary and physical activity data. Anthropometrics, blood samples and dietary intake were collected at baseline and 6 months. A total of 120 [37 CG (age 50.9 \pm 7.1 years; body mass index (BMI) $\left.31.6 \pm 5.8 \mathrm{~kg} / \mathrm{m}^{2}\right), 40 \mathrm{GEP}(\mathrm{age} 42.9 \pm 12.2$ years; BMI $34.8 \pm 9.0 \mathrm{~kg} / \mathrm{m}^{2}$ ) and 43 WEP (age $43.7 \pm 8.1$ years; BMI $30 \pm 5.1 \mathrm{~kg} / \mathrm{m}^{2}$ )] participants completed the study.

Results: Haemoglobin A1c (HbA1c; primary endpoint) significantly improved in all groups over time, with no difference in between-group comparisons. Between group comparisons adjusted for age revealed a clinically significant reduction in BMI in favour of GEP $(p=0.02)$ post-intervention. A clinically significant reduction was also observed in favour of GEP in terms of weight ( $p=$ $0.003)$, waist circumference $(\mathrm{p}=0.017)$, systolic and diastolic blood pressure $(\mathrm{p}$-values $<0.01)$, triglycerides $(\mathrm{p}<0.001)$ and caloric intake $(\mathrm{p}<0.005)$ over time.

Conclusion: Prediabetes education programs of 6-month duration, whether delivered through an intensive lifestyle modification, social media or standard care, are equally efficacious in improving HbAlc levels among Saudi women with prediabetes, but intensive lifestyle is superior in terms of weight reduction and over-all cardiometabolic improvement.

\section{Conflict of Interest}

"There is no conflict of interest" 